\title{
114 VISUALIZATION OF OIL FILM BEHAVIOR ON FRICTION PLATE IN WET CLUTCH ENGAGEMENT
}

\section{J. J. GIL*, H. SUGIYAMA*, T. ARAI*, N. TAKAKURA**, J. TAKAHASHI**}

\begin{abstract}
Visualization study of the behavior of lubricating oil film between rotating wet clutch disks used in automatic transmissions, taking account of the porous facing of one disk, was conducted by a high speed video camera when the plates were engaging. Pressure on porous surface of the plate, friction torque acted between disks, and rotating speed of the reaction plate were measured simultaneously with the visual recording. It was found that air bubbles became visible on surface of porous plate during the boundary lubrication stage. The variation of surface pressure had a strongly effect on the behavior of air bubbles.
\end{abstract}

\section{Introduction}

Squeeze-film prevents the wet clutch disks from making instantaneous contact; and at the completion of the engagement, a considerable sharp rising in the pressure on the disk's surfaces takes place within a relatively short period of time, with torque capacity determined by both clamping force and static frictional coefficient. Although the presence of bubbles inside the lubricant film might affect the performance significantly, there is still much to be learned about the incidence and location of this phenomenon in actual coupling systems, whereas it is one of the most important unknowns in the study of wear and lubrication. The present investigation primary purpose is concerned with the bubbles' behavior all over the porous friction facing; and it differs from previous efforts in literature, since both pressure data achievement and visual recording were conducted in the same friction samples, at the same location, and simultaneously. Comparison of visual and pressure data provided considerable insight into the possible occurrence of cavitation, adding a valuable information about the mechanism of lubrication, in the sense that the bubbles' presence, detrimental to the lubrication effectiveness, has been experimentally confirmed; and from now on, they should be thoroughly taken into consideration.

\section{Testing Apparatus and Specimen}

In order to allow high-speed visual operation and measurement of pressure in synchronization, several modifications were made of the test rig adapted specifically for investigations by scanning type infrared thermograph 1); an outline of relative positions of apparatus, basic instrumentation arrangement and test set-up used to capture high-speed video images, is depicted in Fig. 1.

Dimensions are given in Fig. 2, which shows a schematic diagram of the special test specimen for the specific propose of pressure detection. Outer and inner diameters of the sample lined on the steel axle core were $56 \mathrm{~mm}$ and $10 \mathrm{~mm}$, respectively. It was put to use the same layer of paper friction material of $1 \mathrm{~mm}$ in thickness, throughout the whole study. A thin epoxy boot pressed fit into the facing, provided a $0.5 \mathrm{~mm}$ oil entrance to the cavity where was set the sub-

* Dept. of Fluid Mechanics; Muroran Institute of Technology ** R. \& D. Dept.; DYNAX Corp.

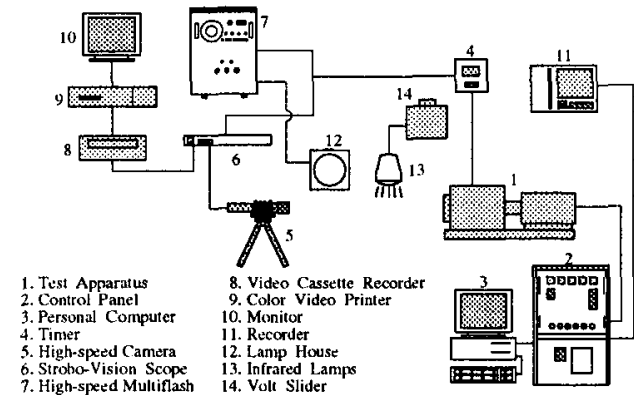

FIG. 1 : Arrangement of Visual Observation Test Rig

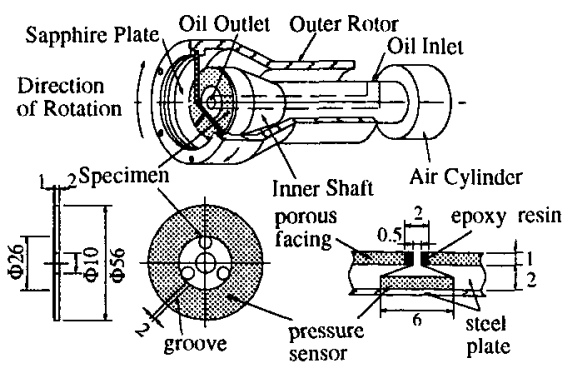

FIG. 2 : Details of Apparatus, Specimen and Transducer 
miniature dynamic pressure transducer, of $6 \mathrm{~mm}$ diameter. Moreover, a $2 \mathrm{~mm}$ width oil groove was formed on the paper friction surface of the test specimen, permitting fluid circulation during coupling progression.

On this rig, apart from contact oil pressure, sliding velocity, piston applied load and torque of friction, were taken also as parameters and recorded continuously, just in a similar way to that operated commonly 1). All the tests were run at approximately the same oil inlet temperature.

\section{Experimental Procedure}

Sapphire and specimen friction plates were cleaned and installed in the friction tester chamber, and the pressure transducer's cavity was repeatedly filled with lubricant before the tests, to extract the air from it. Thrusting load device was aligned by way of a non-rotating physical testing, with pressure sensitive paper. An applied load of $193 \mathrm{kgf}(425.49 \mathrm{~N})$ and three whirl speeds, viz. $2000 \mathrm{rev} / \mathrm{min}(209.44 \mathrm{rad} / \mathrm{s}), 3000$ $\mathrm{rev} / \mathrm{min}(314.16 \mathrm{rad} / \mathrm{s})$ and $4000 \mathrm{rev} / \mathrm{min}(418.88 \mathrm{rad} / \mathrm{s})$, were used in the experiment. During the overall testing presented, the high-speed motion analyzer video-camera and image's processor were operated at 250 , 500,4500 or 9000 frames per second, with a shutter speed of 1/4500 (three firsts) and 1/9000 (latest case) seconds. The most representative pictures were provided by a video printer attached to the video deck.

\section{Test Results and Discussion}

A general observation about test sample' behavior is illustrated in the visual set of Fig. 3, including the result of pressure registration, which indicates a similar tendency as was previously reported2). The region where the pressure acquired a peak value was regarded as representing the abrupt shift from hydrodynamic lubrication to boundary lubrication. Additional oscillatory undulations were detected in the form of distortion, supposed to be brought about from the asymmetry and non-parallelism existing among friction sample and sapphire plate; this undesirable alignment error was minimized by the previous static pressure examination.

It has been distinguished the following situations as the most significant:

- Initially, it was noted the full lubrication film stage before the air cylinder thrusting pressure, when the high velocity of rotation and modes of operation for wet type of clutches made them quite susceptible to air entrainment, which would partially dissolve into the fluid solution (thought to be depending on the applied pressure), or creep under the lumps of inlet flow; it affected the oil extension ratio and prompted a short of instability, with a certain prevention of lubrication over the specimen's surface 3 ). Illustration (a) exhibits this floating layers' effect.

- Picture (b) represents the squeeze film process, which led to the formation of a homogeneous mixture of air and oil, spreading all over the disc' porous facing.

- Then, it turned into the boundary lubrication regime with a steep decrease in oil pressure, and an interesting white area was perceived as seen in (c), indicating the progressive extension of the separation zone or the gradual rupture of the continuous film, with seepage of fluid away out from the porous matrix 4 ); thus, it related with the clearances where the attachment of discs was performed, with most of the engagement pressure sustained by the asperity contact. On the other hand, the observed darker region corresponds to the load carrying zone, in which the whole nonconnecting gap remained filled with a coherent oil film.

The time initiation of each operation was fixed to the moment the air cylinder started pushing the sample disc, forcing

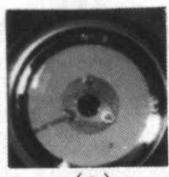

(a)
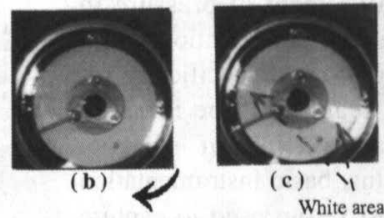

Rotation sense PRESSURE (kPa)

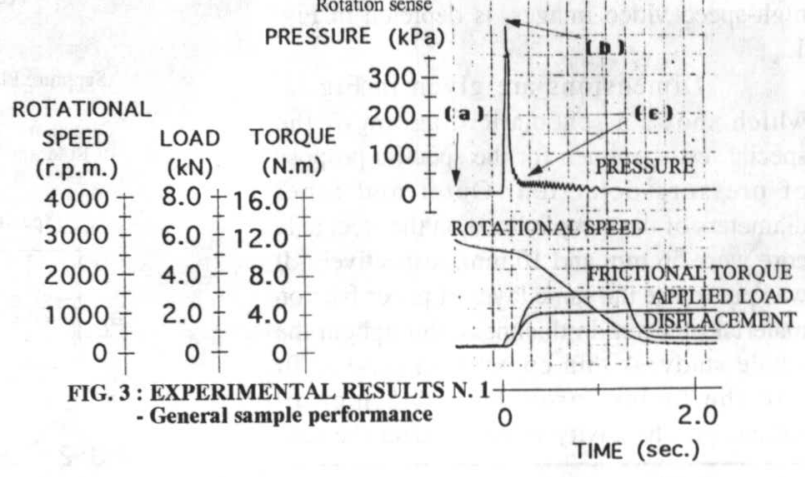


consequently the approach between both of them. It is important to comment the no influence on torque transference during the squeeze-film period, whereas the registered torque became effective from the moment in which the boundary regime and friction coupling, took place. Since it did exist a displacement to be run for the air propelling piston, an evident retardation time in the thrust load evaluation was graphically revealed.

The series of video-prints displayed in Fig. 4, outline a more specific appreciation of the phenomena at the partial view of the fluid, in the upper part of the specimen, for a speed of $4000 \mathrm{rpm}$. Outgrowth of a cloud of bubbles, probably due to the imperfect venting of the test chamber above explained, prompted to a possible availability of cavitation nuclei in the mixture while the progression of the engagement, which presumably reduced tensile strength, and therefore, load-carrying capacity of the oil film. As observed from picture (c) through (f), their amount and size did seem to receive a negligible increase while the run was going on.

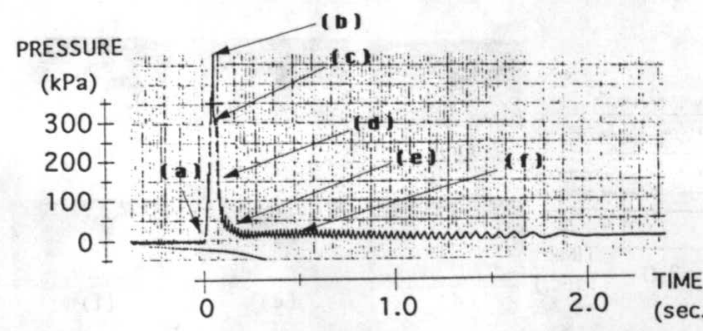

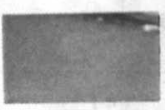

(a)

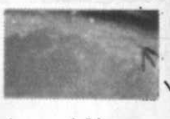

(d)

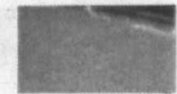

(b)

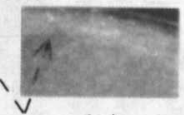

(e)

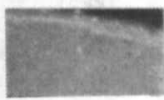

(c)

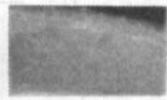

(f)

FIG. 4 : EXPERIMENTAL RESULT N. 58 : Upper disc's region at 4000 r.p.m.

Several frames of pictures that were extracted from the movies, concerning particularly with the typical fluid flow patterns in the sensor region, at whirling velocities of 2000, 3000 and $4000 \mathrm{rpm}$, are shown from Fig. 5 through Fig. 7. As it was formerly hypothesized for the hydrodynamic lubrication stage, the region observed in (a) contained a two-phase mixture of oil and air.

The influence of shaft's whirl velocity was such that the film rupture extension, the number of bubbles and the cavitation effect increased according to the ascent in speed of rotation, as understood on comparing the top peak of the registered pressure (may be due to a significant retrogression in the amount of oil film according to the assumed centrifugal acceleration), and/or the views designated as (b) in each one of the displayed figures. Occasionally, some few bubbles were present in the test chamber as noticed in the illustrations (c), and these bubbles appeared not to be synchronous with speed since they were visualized in a similar location for the three different rotation velocities.

The cavitation bubbles could contain oil vapor and most likely also air, and their existence could have been, respectively, caused by the drastic reduction in oil pressure and the fact of no thoroughly degassification of the test chamber5).

Sometimes, the cavitation bubble could shrink in size during the compression portion of the test, or be transported and still persist encompassing the residual filaments of the fractured oil film, as demonstrated in picture (d) of Fig. 7. Most of times, however, they dissolved or collapsed leaving only a few small bubbles or just air, as perceived in the other figures. The effect of pressure fluctuations could be commented from the set formed by video-prints (e) and (f) contained in these three figures, showing that the fluid flow in the porous matrix was significantly affected by the oil pressure under boundary lubrication conditions, since existed inflow or outflow of the lubricant across the interface between the frictional surface clearance and the porous matrix, according to the pressure gradient positive or negative, respectively4). Moreover, the amount of fluid dragged into the converging clearance through the porous matrix increased as the shaft spinned, because of the inertia effect, inducing thereby a build-up positive hydrodynamic oil-feed pressure that sucked the flow into the gap, where the oil film pressure would be relatively small or negative most probably caused by viscous shear forces. The oil flow into the clearance was more restricted towards the radium medium area, as a result of its larger pressure drop across the porous matrix, (called as "pumping effect"). The transient oil distributed itself through the contact where the oil had cavitated, ruptured or separated, and clearly it did not carry any of the load, as occurred under hydrodynamic lubrication conditions. It was recognized the difference of contact condition caused by the delicate ununiformity at the sliding surface. 


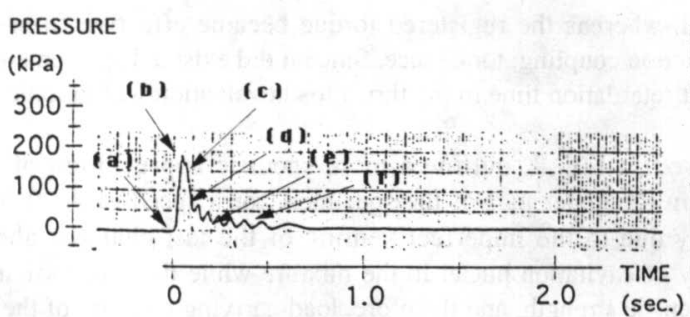

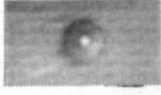

(a)

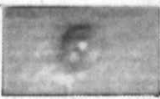

(d)

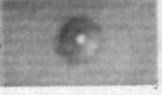

(b)

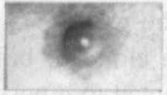

(e)

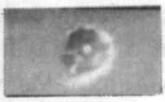

(c)

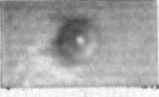

(f)

FIG. 5 : EXPERIMENTAL RESULT N. 44 : Pressure sensor region at 2000 r.p.m.

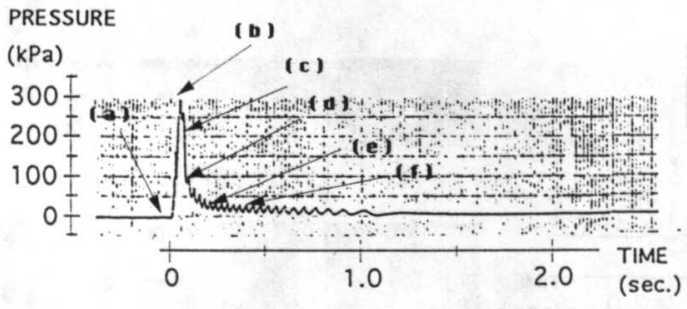

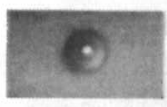

(a)

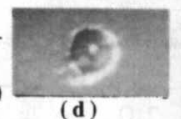

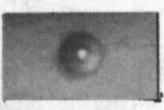

(b)

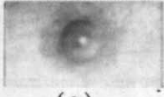

(e)

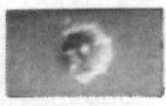

(c)

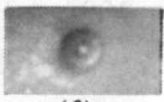

(f)

FIG. 6 : EXPERIMENTAL RESULT N. 32: Pressure sensor region at 3000 r.p.m.

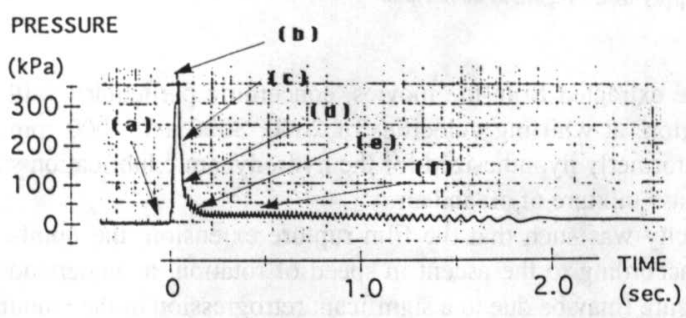

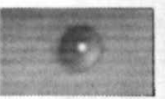

(a)

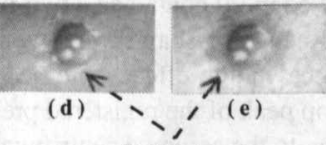

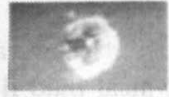

(c)

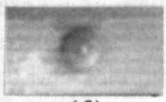

(f)

FIG. 7 : EXPERIMENTAL RESULT N. 49: Pressure sensor region at 4000 r.p.m. Bubbles

\section{Concluding Remark}

To the author's knowledge, it does not exist any comparable study in relation to the wet clutch coupling mechanism, including expressly the simultaneous ability of both visualization and pressure registration in the analysis of wet clutch engagement; in order to add some more light about cavitation's influence, mixture compressible properties should be further examined.

\section{References}

1 Ohnuma, H., and Kato, K., "The Effect of Groove Pattern of Paper Friction Plate on Its Life", SAE Paper, No. 910804, 1991.

2 Ito, H., Fujimoto, K., Yamamoto, T., and Yamagishi, N., "Measurement of Oil Film Pressure During the Movement Process, from the Facing Material Used in Wet Clutch", Proc. of Tribology Conference, JST., Nagoya, Nov. 1993, pp. 673 - 676, (in Japanese).

3 Nakahara, T., Yamazaki, T., and Kyogoku, K., "Prevention of Oil Feeding by Air Film Formation", Proc. Japan International Tribology Conference, 1990, pp. 1623 - 1628.

4 Kaneko, S., Inoue, H., and Ushio, K., "Experimental Study on Mechanism of Lubrication in Porous Journal Bearings (Oil Film Formed in Bearing Clearance)", JSME International Journal, Series C, Vol. 92, 1994, pp. $185-192$.

5 Sun, D.C., Brewe, D.E., and Abel, P.B., "Simultaneous Pressure Measurement and High-Speed Photography Study of Cavitation, in a Dynamically Loaded Journal Bearing", ASME Journal of Tribology, Vol. 115, Jan. 1993, pp. 88 - 95. 